\title{
Study of the Performance of a New Kind of Downhole Gas-Liquid Separation with High Gas Content
}

\author{
Fanchen Meng ${ }^{1}$, Shiying $\mathrm{Shi}^{2}$, Naiqing $\mathrm{Ma}^{2}$ \\ ${ }^{1}$ Oil \& Gas Cooperation and Development Company, BHDC, Neimenggu Eerduosi, China \\ ${ }^{2}$ Institute of Mechanics, Chinese Academy of Sciences, Beijing, China
}

Email address:

shishiying@imech.ac.cn (Shiying Shi)

\section{To cite this article:}

Fanchen Meng, Shiying Shi, Naiqing Ma. Study of the Performance of a New Kind of Downhole Gas-Liquid Separation with High Gas Content. Journal of Energy and Natural Resources. Vol. 8, No. 2, 2019, pp. 45-49. doi: 10.11648/j.jenr.20190802.11

Received: January 7, 2019; Accepted: May 8, 2019; Published: May 23, 2019

\begin{abstract}
In the downhole, gas separation is crucial for the Electrical Submergible Pumps' normal operation. This paper presents a new type of downhole gas-liquid separator (DGLS) and studies its separation performance in experiments that interests the oil industry. Laboratory results show that the pressure drop in the DGLS is rather small and that the gas-liquid separation in the DGLS can be seen as an incompressible flow. When the split ratio equals the gas content at the inlet, complete gas-liquid separation occurs. In addition, as the liquid density increases, the gas-liquid separation performance improves. The separation mechanism of the DGLS is related to the centrifugal acceleration, which is better when it is less than 30 times the gravitational acceleration. A method for predicting the separation performance is proposed.
\end{abstract}

Keywords: Downhole Gas-Liquid Separation, Split Ratio, Gas Content, Centrifugal Acceleration, Experiment

\section{Introduction}

During gas extraction, as the gas pressure and gas flow rate gradually decrease, the output of gas reservoir water or liquids cannot be carried out of the wellbore by the natural air flow and is thus stranded in the wellbore [1-5]. These liquids accumulate over a period of time in the bottom hole to form a fluid column, which causes additional hydrostatic pressure in the reservoir. Under this condition, the gas well energy flow declines [6-9]. Eventually, the accumulated fluid column in the wellbore will cause the gas well to stop production [10-13].

To solve the above problems, the bulky conventional gravity-based vessel-type separator cannot be used in downhole [14]. Thus, a new kind of axial Down-hole Gas-Liquid Separator (DGLS), as shown schematically in Figure 1, is proposed and studied in this paper. The DGLS is a pipe that covers half of the cylinder pipe, with bi-symmetric involute-shaped entrances between them. The phases enter the cylinder pipe through bi-symmetric involute-shaped entrances and move in a swirling motion. In the DGLS, the swirling produces a centrifugal separation, whereby gas moves toward the pipe central area and exits through the top (the gas outlet) and a water-rich stream leaves the system through the liquid outlet due to gravity.

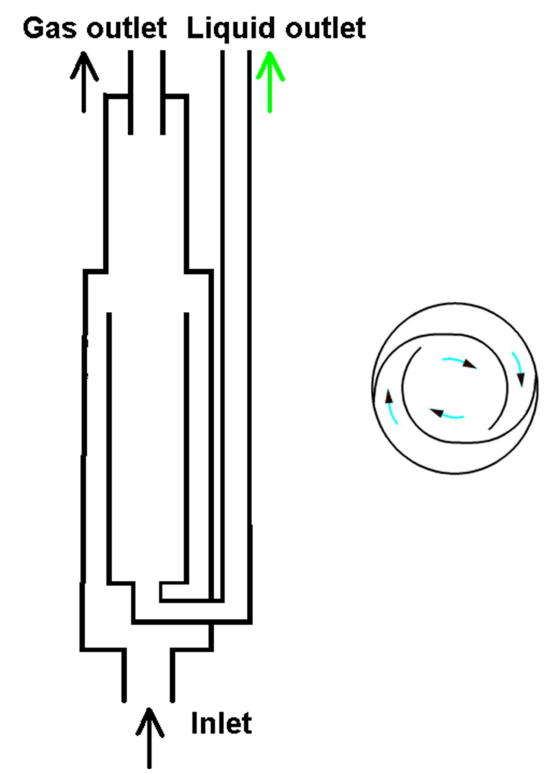

Figure 1. Schematic representation of the DGLS and cross section at its bi-symmetric involute shaped entrances. 


\section{Method}

\subsection{The Structure of the DGLS}

To replicate the separation process of the DGLS described above, an experimental DGLS is constructed at the Laboratory of Applied Fluid Dynamics in CAS, and experiments of gas-liquid flow inside the DGLS are conducted. The DGLS in Figure 2 is made of plexiglass to enable visual observation of the gas-liquid separation process. The DGLS is an $800 \mathrm{~mm}$, $100 \mathrm{~mm}$ ID vertical pipe covering a $750 \mathrm{~mm}, 60 \mathrm{~mm}$ ID cylindrical pipe. A $15 \mathrm{~mm}$ ID pipe located at the top is used as the gas outlet, and the liquid outlet is a $25 \mathrm{~mm}$ ID pipe located at the bottom.

\subsection{Flow-loop}

In the experiments, the gas is mixed with the liquid with a mixer, and the gas/liquid mixture is separated by the DGLS and enters the sampling bucket. Finally, gas is released, and the liquid flows back to the water tank. In this study, air and water are used in the experiments, and the physical properties are as follows $\left(20^{\circ} \mathrm{C}\right)$ :

Table 1. Physical property of the experimental liquids.

\begin{tabular}{lll}
\hline & Density $\left(\mathrm{kg} / \mathbf{m}^{\mathbf{3}}\right)$ & Viscosity $(\mathbf{m P a} \cdot \mathbf{s})$ \\
\hline Water & 998.2 & 1.00 \\
Gas & 1.2 & $1.81 \times 10^{-3}$ \\
\hline
\end{tabular}

Pressure sensors are located at each outlet to calculate the pressure drop. Two valves are installed vertically at the gas outlet and the water outlet to calculate the gas volume fraction and the control of the flow rates leaving the DGLS.

The gas volume fraction $\alpha_{g}$ is defined as

$$
\alpha_{g i}=\frac{Q_{g i}}{Q_{g i}+Q_{l i}}
$$

where $Q_{g i}$ is the gas flow rate, $Q_{l i}$ is the liquid flow rate, and the subscrips 1-3 refer to the inlet, the gas outlet and the liquid outlet.

The liquid volume fraction $\alpha_{l}$ is defined as

$$
\alpha_{l i}=1-\alpha_{g i}
$$

In the experiments, the gas volume fraction at the inlet is controlled between 50.0 and $95.0 \%$.

\section{Results}

The measured static pressure at the inlet and the pressure drop between the inlet and two outlets are shown in Table 2. The experimental results show that the pressure at the inlet is higher than those at the gas and liquid outlets. The pressure drop between the inlet and the two outlets is less than $5 \mathrm{kPa}$, and the inlet pressure is greater than $100 \mathrm{kPa}$.

Table 2. The experimental results about pressure.

\begin{tabular}{llllll}
\hline $\boldsymbol{Q}_{\boldsymbol{I}}\left(\mathbf{m}^{\mathbf{3}} / \mathbf{d}\right)$ & $\boldsymbol{\alpha}_{\boldsymbol{g} \mathbf{1}}$ & $\mathbf{p}_{\mathbf{I}}(\mathbf{P a})$ & $\left.\boldsymbol{\Delta}_{\mathbf{1 3}} \mathbf{( P a}\right)$ & $\boldsymbol{\Delta}_{\mathbf{1 2}}(\mathbf{P a})$ & $\boldsymbol{\alpha}_{\boldsymbol{g} \mathbf{3}}$ \\
\hline 69 & 0.65 & 103039 & 1434 & 808 & 0.01 \\
69 & 0.65 & 102572 & 1092 & 1024 & 0.00 \\
145 & 0.63 & 103490 & 2212 & 1254 & 0.18 \\
145 & 0.63 & 103382 & 2157 & 1232 & 0.14 \\
145 & 0.63 & 103366 & 2122 & 1244 & 0.02 \\
145 & 0.63 & 103361 & 1919 & 1714 & 0.00 \\
210 & 0.84 & 104466 & 1737 & 869 & 0.30 \\
210 & 0.84 & 104327 & 1705 & 912 & 0.12 \\
210 & 0.84 & 104306 & 1650 & 930 & 0.10 \\
210 & 0.84 & 103588 & 1479 & 976 & 0.05 \\
210 & 0.84 & 105719 & 1451 & 1135 & 0.00 \\
289 & 0.92 & 105621 & 1305 & 797 & 0.50 \\
289 & 0.92 & 105261 & 1239 & 1078 & 0.00 \\
\hline
\end{tabular}

According to the PVT equation of state, the following relationship can be obtained. As can be seen, the absolute volume change rate is less than 0.05 , which means that the liquid in the DGLS is nearly incompressible.

$$
\left|\frac{\Delta V}{V}\right| \approx \leq\left|-\frac{\Delta p}{p}\right| \leq 0.05
$$

where $\mathrm{V}$ is the fluid volume in the DGLS, $\Delta V$ is the fluid volume change in the DGLS.

The experimental results suggests that the split-ratio can be introduced to describe the gas-liquid separation in the DGLS. The split-ratio is defined as the ratio between the liquid outlet flow rate and the inlet flow rate and is given by

$$
F=\frac{Q_{W}}{Q_{I}}
$$

where $Q_{W}$ is liquid outlet flow rate, $Q_{I}$ is the inlet flow rate.

\section{Discussion}

Figure 2 shows the effect that the split ratio has on the DGLS' separation performance when the velocity is $0.88 \mathrm{~m} / \mathrm{s}$ and the gas content is 0.78 at the inlet. As shown, when the split ratio increases, the gas content at the water outlet increases, and the water content at the gas outlet decreases. There is an optimal split ratio that equals the liquid content at the inlet exists for gas-liquid separation in the DGLS because the gas and water in the DGLS form a weak swirl flow under the action of gravitational and centrifugal forces. A gas-liquid interface that looks like a bowl is produced in the weak swirl flow. When the split ratio increases, the gas-liquid interface decreases to the water outlet, and the gas content at the water outlet increases. When the split ratio equals the gas content at the inlet, the gas and water are fully separated: the gas content at the gas outlet is $100 \%$, and that at the water outlet is $0 \%$. 


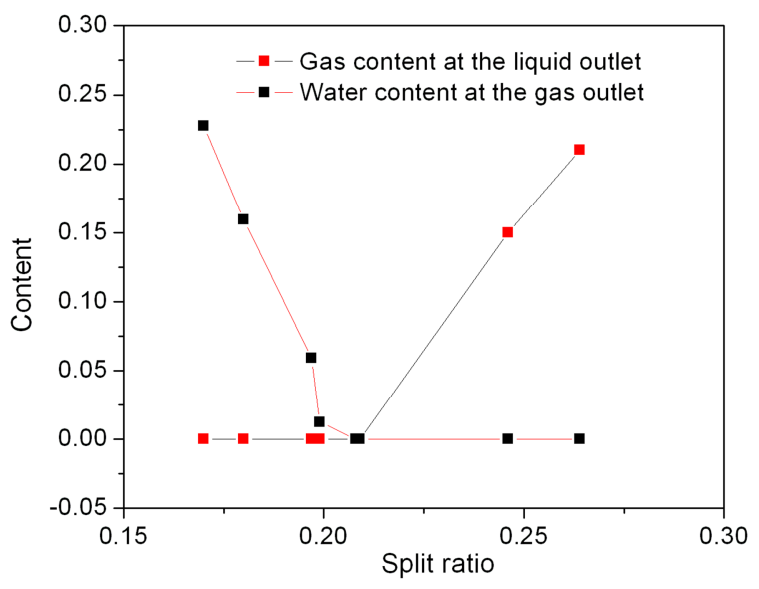

(a) Influence of the split ratio on two outlet separation performance

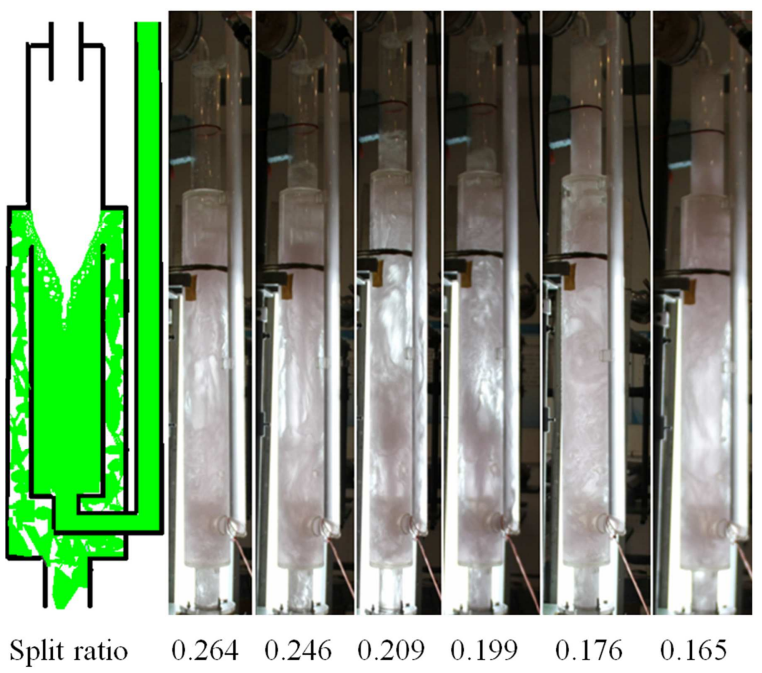

(b) Gas-liquid interface evolving with decrease in the split ratio

Figure 2. Effect of the split ratio on the phase separation performance.

When the mixture velocity is $0.85 \mathrm{~m} / \mathrm{s}$ at the inlet, the influence of the gas content on the gas-liquid separation performance of the DGLS at the inlet is shown in Figure 3. It is shown that complete phase separation is achieved for any gas content at the inlet as long as the split ratio approximately equals the liquid content at the inlet.

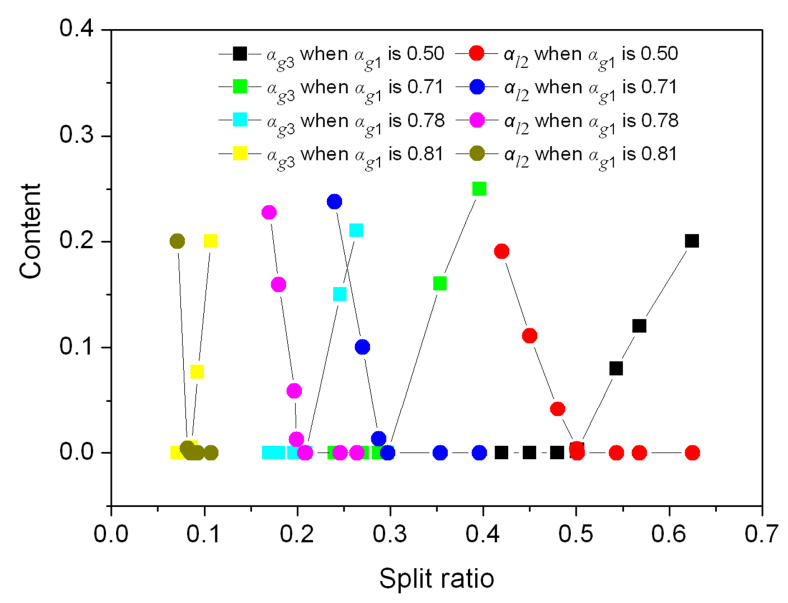

Figure 3. Gas-liquid separation at different inlet gas content.
Figure 4 shows the effect of the fluid viscosity on the gas-liquid separation when the gas content is 0.63 and the mixture velocity is $0.85 \mathrm{~m} / \mathrm{s}$ at the inlet. It can be seen that when the liquid viscosity increases, the gas content at the liquid outlet increases and the liquid content at the gas outlet changes slightly. This is because when the liquid viscosity increases, the drag on the gas bubbles required for them to separate from the viscous liquid becomes larger. Thus, more bubbles are retained in the liquid, and the gas content at the liquid outlet increases. The drag on the liquid droplets generated in gas changes only slightly, so the liquid content at the gas outlet does not change excessively.

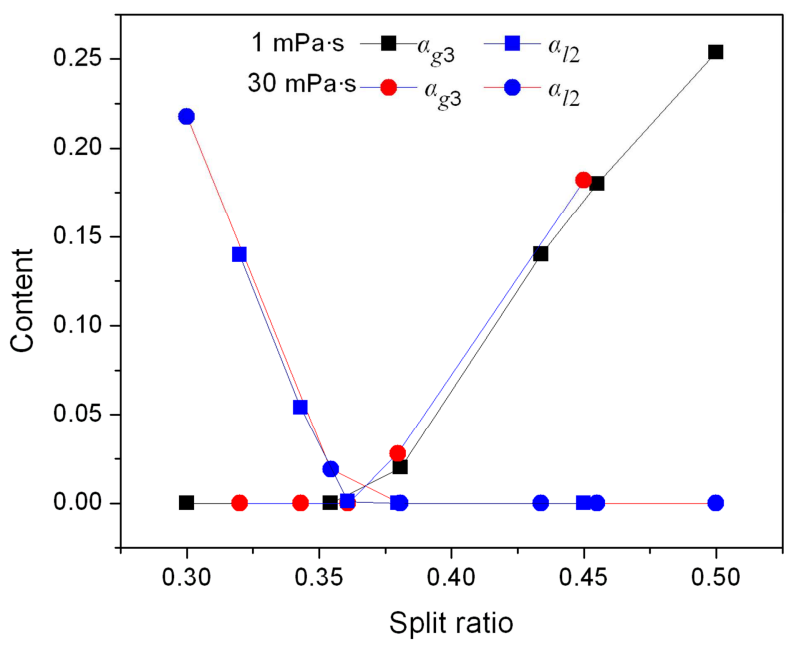

Figure 4. Effect of liquid viscosity on performance.

The design of the DGLS is based on the concept of gravitational and centrifugal separation. Thus, the limits of centrifugal acceleration are critical to the design of the DGLS. The centrifugal acceleration $a$ is defined as

$$
a=\left(\frac{Q_{1}}{2 L H}\right)^{2} /(R g)
$$

where $\mathrm{L}$ and $\mathrm{H}$ is the bi-symmetric involute-shaped entrances' width and height; $\mathrm{R}$ is the average distance from the entrances' center to the cylinder's axis.

Figure 5 shows gas-liquid separation in the DGLS when the gas content at the inlet is 0.63 and the centrifugal acceleration changes. When the centrifugal acceleration is low, liquid and gas are mainly separated by gravity. Under this condition, the liquid locates at the bottom of the cylinder space, and the gas distributes in the upper part. Complete phase separation occurs in the DGLS. When the centrifugal acceleration increases, a gas core shaped like a fine cone forms. If the centrifugal acceleration is too large, the gas core will expand to fill the whole cylinder. Under this condition, incomplete separation occurs, which means that liquid flows out through the gas outlet and gas leaves the DGLS through the liquid outlet, with liquid. Experiments show that the critical centrifugal acceleration is within 30 times that of gravity, which means that gravity plays a major role compared to centrifugal force. 


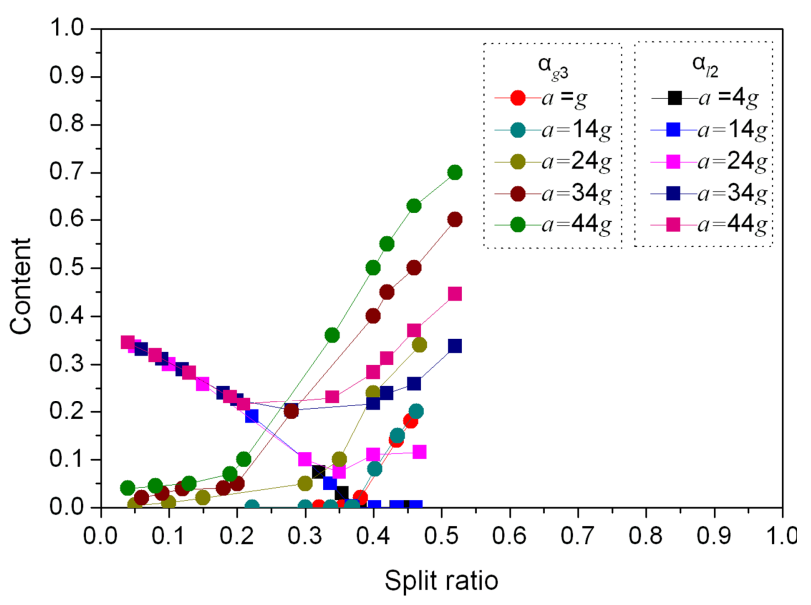

Figure 5. Influence of centrifugal acceleration on gas-liquid separation in the $D G L S$.

When gravity's effect is non-negligible in gas-liquid separation, the gas content at the liquid outlet can be calculated by the following method

$$
\begin{gathered}
\alpha_{g 3}=\frac{F-\alpha_{g 1}}{F} \quad\left(F>\alpha_{g 1}\right) \\
\alpha_{g 3}=0 \quad\left(F \leq \alpha_{g 1}\right)
\end{gathered}
$$

The gas-liquid separation performance can be inferred from the experimental data by Eqs. (6) and (7). The values of the gas-liquid separation experimental results obtained when gravity plays a major role are compared with those from the inferred model and shown in Figure 6. As can be seen, the results predicted are very satisfactory. They demonstrate that the method can be used to calculate the gas content at the liquid outlet for gas-liquid separation when gravity plays an important role [15].

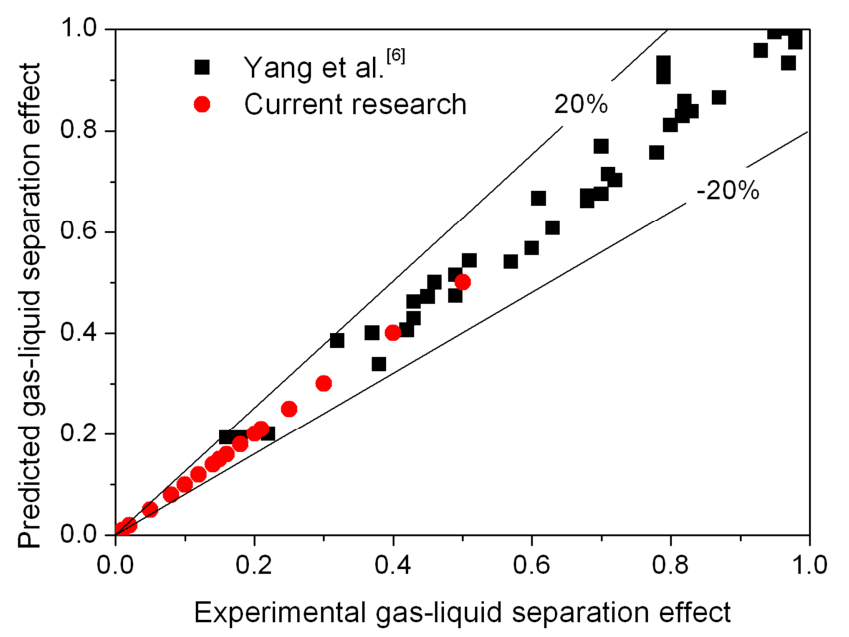

Figure 6. Comparison between experimental and predicted separation performance.

\section{Conclusion}

An experimental investigation of the gas-liquid separation in a DGLS is carried out when gas content is greater than $50 \%$. The separation performance of the DGLS is studied experimentally. The pressure drop in the DGLS is small, and the gas-liquid flow can be considered an incompressible flow. When the gas content at the inlet changes, an optimal split ratio exists and approximately equals the liquid content at the inlet. When the liquid viscosity increases, the gas content at the liquid outlet increases, and the liquid content at the gas outlet does not change significantly. For DGLS, the centrifugal acceleration is better when it is within 30 times the gravitational acceleration, and a method for predicting the gas content in the liquid is derived.

\section{References}

[1] Carios E., Vega L., Pardo R., et al. Experimental Study of a Poor Boy Downhole Gas Separator Under Continuous Gas-Liquid Flow [M]. 2013.

[2] Hreiz R., Lainé R., Wu J., et al. On the effect of the nozzle design on the performances of gas-liquid cylindrical cyclone separators [J]. International Journal of Multiphase Flow, 2014, 58 (1): $15-26$.

[3] Ortiz-Vidal L. E., Barbosa M. C., Rodriguez O. M. H.. High efficiency gas-liquid separation system for pumped wells $[\mathrm{J}]$. Petroleum, 2018.

[4] Movafaghian S., Jaua-Marturet J. A., Mohan R. S., et al. The effects of geometry, fluid properties and pressure on the hydrodynamics of gas-liquid cylindrical cyclone separators $[\mathrm{J}]$. International Journal of Multiphase Flow, 2000, 26 (6): 999-1018.

[5] Lucero D. P.. A downhole gas separator for methane production from geopressured aquifers [J]. Applied Energy, 1984, 16 (3): 193-221.

[6] Liu X. L., Falcone G., Teodoriu C.. Liquid loading in gas wells: Experimental investigation of back pressure effects on the near-wellbore reservoir [J]. Journal of Natural Gas Science and Engineering, 2016, 36: 434-441.

[7] Ortiz-Vidal L. E., Barbosa M. C., Rodriguez O. M. H.. High efficiency gas-liquid separation system for pumped wells [J]. Petroleum, 2018: 1-5.

[8] Weingarten J. S., Kolpak M. M., Mattison S. A. et al. Development and testing of a compact liquid-gas auger partial separator for downhole of surface applications. SPE Production and Facilities, 1997: 34-40.

[9] Wissink J. G.. DNS of separating low Reynolds number flow in a turbine cascade with incoming wakes. International Journal of Heat and Fluid Flow, 2003, 24 (4): 626-635.

[10] Rollet-Miet P., Laurence D., Ferziger J.. LES and RANS for turbulent flow in tube bundles. International Journal of Heat and Fluid Flow, 1999, 20 (3): 241-254.

[11] Jack F. M.. Aerospace gas-liquid separator for terrestrial applications. IEEE, 1996: 109-113.

[12] Weomgarten J. S., Kolpak M. M., Mattison S. A. et al. Development and testing of a compact liquid -gas auger partial separator for downhole or surface applications. SPE Production and Facilities, 1997: 34-40. 
[13] Bohorquez R., Ananaba V., Alabi O. et al. Laboratory testing of downhole gas separators [J]. SPE Production and Operations, 2009, 24 (4): 499-509.

[14] Enrique C., Libar V., Raimundo P. et al. Experimental study of a poor boy downhole gas separator under continuous gas-liquid flow [C]. SPE Artificial Lift Conference-Americas, 2013, 21-22 May, Cartagena City, Colombia.

[15] Yang L. L., Liu S., Li H., et al. Gas-liquid flow splitting in T-junction with inclined lateral arm [J]. Journal of Hydrodynamics, 2018, 30 (1): 173-176. 